\title{
Additive Decomposition Applied to the Semiconductor Drift-Diffusion Model
}

\author{
ELIZABETH J. BRAUER ${ }^{\mathrm{a}, *}$, MAREK TUROWSKI ${ }^{\mathrm{b}}$ and JAMES M. MCDONOUGH ${ }^{\mathrm{c}}$ \\ ${ }^{a}$ P.O. Box 15600, Department of Electrical Engineering, Northern Arizona University, \\ Flagstaff, AZ 86011-5600; ${ }^{\mathrm{b}}$ Department of Electrical Engineering, University of Kentucky, \\ 453 Anderson Hall, Lexington, KY 40506-0046, USA; ${ }^{\mathrm{c}}$ Department of Mechanical \\ Engineering, University of Kentucky, 514 h Ctr for Robotics \\ and Mfg Systems, Lexington, KY 40506-0108, USA
}

\begin{abstract}
A new numerical method for semiconductor device simulation is presented. The additive decomposition method has been successfully applied to Burgers' and Navier-Stokes equations governing turbulent fluid flow by decomposing the equations into large-scale and small-scale parts without averaging. The additive decomposition (AD) technique is well suited to problems with a large range of time and/or space scales, for example, thermal-electrical simulation of power semiconductor devices with large physical size. Furthermore, AD adds a level of parallelization for improved computational efficiency. The new numerical technique has been tested on the 1-D drift-diffusion model of a $p-i-n$ diode for reverse and forward biases. Distributions of $\phi, n$ and $p$ have been calculated using the AD method on a coarse large-scale grid and then in parallel small-scale grid sections. The AD results agreed well with the results obtained with a traditional onegrid approach, while potentially reducing memory requirements with the new method.
\end{abstract}

Keywords: Numerical methods, semiconductor, simulation, drift-diffusion, decomposition

\section{INTRODUCTION}

The numerical method, additive decomposition, has been successfully applied in mechanical and chemical engineering to Burgers' equation and the Navier-Stokes equations governing turbulent fluid flow by decomposing governing equations into large-scale and small-scale parts without averaging, e.g., [1-3]. The additive decomposition (AD) technique is well suited to problems with a large range of time and/or space scales, for example, thermal-electrical simulation of power semiconductor devices with large physical size. Thermal-electrical effects have a large range of

\footnotetext{
* Corresponding author.
} 
time scales since the electrical time constants are much faster than the thermal time constants. Furthermore, additive decomposition adds a level of parallelization for improved computational efficiency. Thus, semiconductor device simulation is a natural application of the additive decomposition numerical technique. Initially, we decompose the simplest device equations, the drift-diffusion model, to test the method. After successful implementation for the drift-diffusion equations, we plan to apply the new approach to the hydrodynamic semiconductor model.

\section{ADDITIVE DECOMPOSITION OF THE DRIFT-DIFFUSION EQUATIONS}

The standard drift-diffusion model of semiconductors consists of the following equations. Poisson's equation is

$$
\nabla^{2} \phi=-\frac{q}{\varepsilon_{s}}\left(p-n+N_{d}-N_{a}\right)
$$

and the continuity equations for electrons and holes are

$$
\begin{gathered}
\frac{\partial n}{\partial t}=\frac{1}{q} \nabla\left[D_{n} \cdot \nabla n-\mu_{n} \cdot n \cdot \nabla \phi\right]-R \\
\frac{\partial p}{\partial t}=-\frac{1}{q} \nabla\left[-D_{p} \cdot \nabla p-\mu_{p} \cdot p \cdot \nabla \phi\right]-R
\end{gathered}
$$

where the symbols have their standard meaning [4].

Additive decomposition of the drift-diffusion semiconductor model proceeds as follows. First, the basic variables, electrostatic potential, electron density and hole density, are divided into largescale and small-scale components:

$$
\phi=\bar{\phi}+\phi^{*}, \quad n=\bar{n}+n^{*}, \quad p=\bar{p}+p^{*} .
$$

here $\bar{\phi}$ is the large-scale component and $\phi^{*}$ is the small-scale component of the potential, and similarly for $n$ and $p$. After substituting Eq. (4) into Eq. (1) through (3), the equations are then decomposed additively into large-scale and smallscale equations:

$$
\begin{aligned}
\nabla^{2} \bar{\phi} & =\frac{q}{\varepsilon_{s}}\left(\bar{n}-\bar{p}-\overline{N_{d a}}\right) \\
\nabla^{2} \phi^{*} & =\frac{q}{\varepsilon_{s}}\left(n^{*}-p^{*}-N_{d a}^{*}\right)
\end{aligned}
$$

where $N_{d a}=N_{d}-N_{a}$, and

$$
\begin{aligned}
& \frac{\partial \bar{n}}{\partial t}= \nabla\left[D_{n} \nabla \bar{n}-\mu_{n} \bar{n} \nabla \bar{\phi}-\mu_{n} \cdot(1-\beta)\right. \\
&\left.\cdot\left(\bar{n} \nabla \phi^{*}+n^{*} \nabla \bar{\phi}\right)\right]-\bar{R} \\
& \frac{\partial n^{*}}{\partial t}=\nabla\left[D_{n} \nabla n^{*}-\mu_{n} n^{*} \nabla \phi^{*}-\mu_{n} \cdot \beta\right. \\
&\left.\cdot\left(\bar{n} \nabla \phi^{*}+n^{*} \nabla \bar{\phi}\right)\right]-R^{*}
\end{aligned}
$$

Equation (5a) is the large-scale Poisson's equation, (5b) is the small-scale Poisson's equation, (6a) is the large-scale current continuity equation for electrons, and (6b) is the small-scale current continuity equation for electrons. The decomposition of the current continuity equation for holes is similar to (6). The additive decomposition is done in such a manner, that Eqs. (5a) and (5b) added together are equivalent to Eq. (1), and in the same way, Eqs. (6a) and (6b) added together result in the original Eq. (2).

The decomposition parameter $\beta$ is a coupling coefficient between large-scale and small-scale solutions $[1,2]$. The parameter $\beta$ controls the large-scale/small-scale interaction. For example, in the limit $\beta=1$, the effect of large scale changes is transferred to the small scale, but not vice versa. In principle, $\beta$ can be assigned any value between 0 and 1 implying nonuniqueness of the decomposition, but Brown et al. [5] have recently demonstrated optimal values for the incompressible Navier-Stokes equations. In the present study, $\beta=1$ has been used.

Decomposition of the variables in the recombination term $R$ requires a special treatment. In the Shockley-Read-Hall expression for recombination-generation, $n$ and $p$ appear in the numerator 
as well as in the denominator. This makes a straight implementation of the additive decomposition impossible. One possible solution to this problem is to calculate the value of the denominator with the $n$ and $p$ values from the previous iteration. Then, treat the denominator as a constant and decompose only the numerator, using the decomposition parameter $\beta$, which will generate both $R^{*}$ and $\bar{R}$. Another possible method is to linearize $R$ by expanding in a Fréchet-Taylor series, and then decompose the basic variables $n$ and $p$ of the linearization in the usual way. In the preliminary tests of the $\mathrm{AD}$ method presented here, the authors used the first method to decompose the recombination term. It is important to note that the decomposition has been designed so that sums of the large and small-scale equations are the original Eqs. (1-3). For linear problems, this represents a straightforward approach, the validity of which is trivial to demonstrate. For problems of the type considered here, the proof is more involved, and we refer the reader to Brown et al. [5] for an indication of what is needed.

For a 1-D simulation, each large-scale equation is solved on the large-scale grid with $N$ points across the device ( $N$ is quite a small number), and the small-scale equations are solved on the smallscale grid over a section of the device centered around a large-scale grid point; see Figure 1. There is a separate set of small-scale equations (for small-

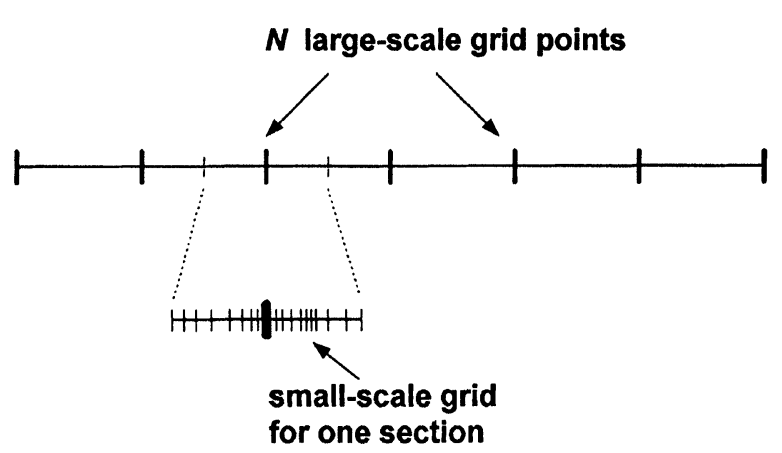

FIGURE 1 Illustration of large-scale and small-scale discretization grid. scale space or time points) for each large-scale grid point. Thus, the small-scale equations can be solved independently and in parallel in $N$ sections. The large-scale solution, which depends on the small-scale solution, combines these to produce total results over the whole simulation domain.

The large-scale function may be defined in various ways between the large-scale grid points. In our first calculations, the large-scale values were set constant within one section, equal to the values obtained from the large-scale solution for the large-scale point of the section; i.e., the large-scale solution representation is piecewise constant when used in the small-scale equations. The small-scale values are variations of the exact solution around the (local) constant large-scale value within one section. The total small-scale function along the entire device length is, of course, not continuous, although the derivative of the small-scale function is the same as the derivative of the total solution where both are well defined. We also tried the approach which is a linear interpolation of the large-scale solution between the large-scale grid points. In this case, the small-scale function is continuous but has discontinuous slope between sections. Nevertheless the convergence of the final solution was much worse with this method than with the first one.

Boundary conditions between the separate sections pose a significant problem, particularly if the sections are computed in parallel. Two types of boundary conditions have been tested, viz., the Neumann type and the Dirichlet type, and it has appeared that properly calculated Dirichlet conditions result in more stable solutions.

\section{NUMERICAL EXPERIMENTS}

As a first test, the new $\mathrm{AD}$ technique has been applied to the 1-D solution of the drift-diffusion transport equations of the $p-i-n$ diode for reversebias and forward-bias conditions. The test $p-i-n$ diode structure is presented in Figure 2. As a computational platform, the PASSC semiconduc- 


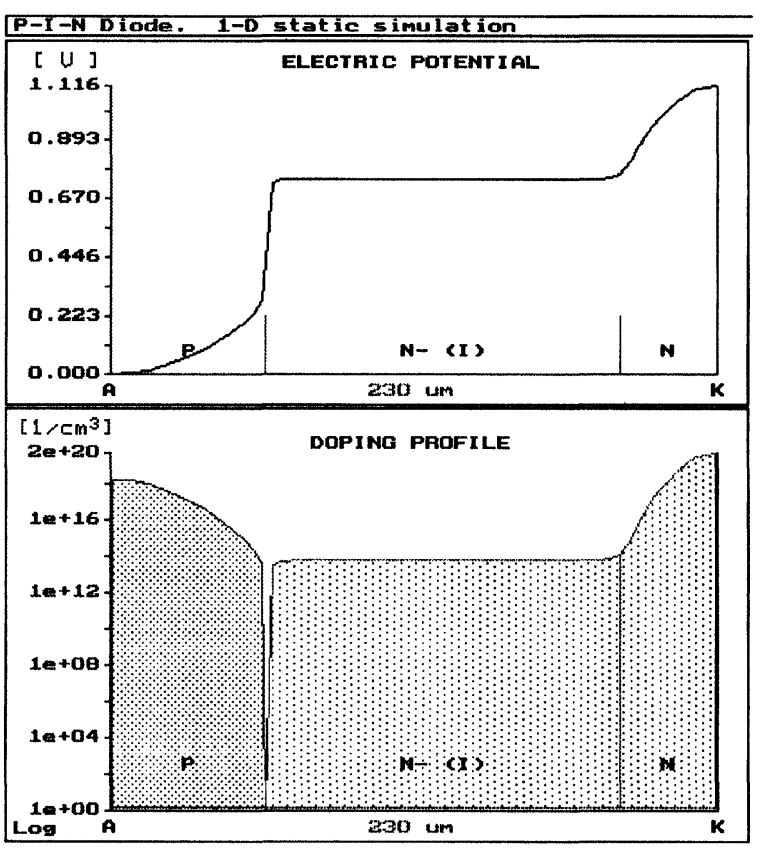

FIGURE 2 Potential distribution for zero bias and doping profile of the best $p-i-n$ diode structure.

tor simulation program [6] has been used, with extensive physical models included [7, 8]. In the present calculations, the decomposition parameter $\beta$ has been set to 1 . The distributions of electrostatic potential $\phi$, electron concentration $n$ and hole concentration $p$ have been calculated as basic results. As a measure of quality of computed results, the space charge formation has been used in the case of zero bias [9] and reverse bias, and the distributions of $\phi, n$, and $p$ as well as current density in the case of forward bias.

First, as a reference, the solution with the traditional method has been obtained, using a non-uniform grid with 40 points in one dimension. Then, a large-scale solution has been computed on $N$ grid points uniformly distributed along the device. In the present study, we have tried $N=9$ and $N=5$. Small-scale results have been calculated in separate sections surrounding each largescale point, except the border ones, which resulted in 7 or 3 separate sections along the device, respectively. With the boundary points of neigh- boring sections overlapping, the sections included from 5 to 12 small-scale grid points for 7 sections, and 15, 9 and 18 small-scale grid points per section in the case of 3 sections. After the large-scale solution has been calculated, the small-scale initial guess values are updated by interpolation using the large-scale solution. Then the accurate small-scale solutions are computed in separate (parallel) sections around each large-scale point. The final results for the forward bias $V_{A K}=1.0 \mathrm{~V}$, calculated with the large grid of $N=5$, are shown in Figure 3. These results are very close to the reference results obtained with the standard onegrid method. The biggest problem in this approach is setting up appropriate boundary conditions between sections, because the final results are very sensitive to the slightest change in boundary conditions of each section. The current density
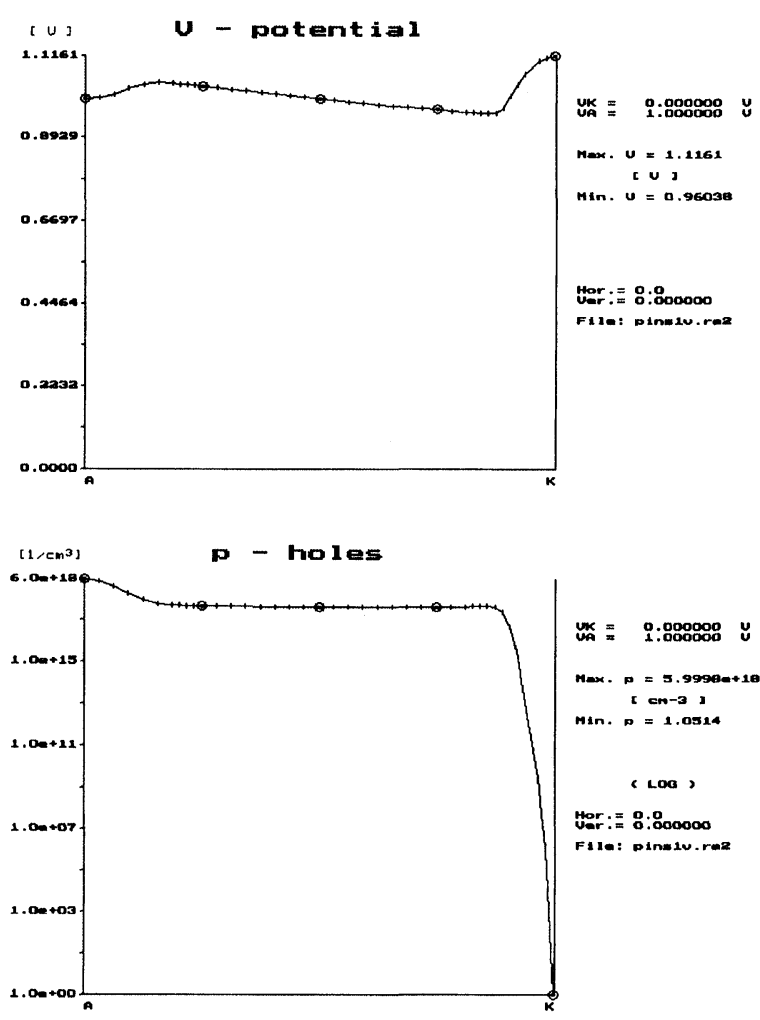

FIGURE 3 Final additive decomposition solution for the forward bias $V_{A K}=1.0 \mathrm{~V}$ the thicker grid lines (circled) correspond to the large-scale grid, $N=5$. 
from the reference results for $V_{A K}=+1.0 \mathrm{~V}$ was 134.7 A/ $/ \mathrm{cm}^{2}$. The current density calculated using only the large-scale solution was $168.4 \mathrm{~A} / \mathrm{cm}^{2}$ for $N=5$ (error $25 \%$ ), and $135.8 \mathrm{~A} / \mathrm{cm}^{2}$ for $N=9$ (error $0.8 \%$ ), and the values were uniform along the entire device length. After solving the smallscale equations in each section, the final current density values obtained with the AD method were slightly different in different sections (within 10\%), which was due to inaccurate small-scale boundary conditions for separate sections. Iterating the small-scale results to recalculate small-scale boundary conditions and re-solving small-scale equations improved the uniformity of the resulting current density, although the rate of improvement was slow.

In a similar manner, results for the reverse bias were calculated. As mentioned already, in this case, the space charge formation has been used as a measure of accuracy of results. Figure 4 shows the net charge $\left(Q=p-n+N_{d}-N_{a}\right)$ calculated on a non-uniform single grid (40 points in 1-D) with the traditional method for the reverse bias $V_{A K}$ $=-1.0 \mathrm{~V}$. The net charge distribution calculated only with the coarse large-scale grid $(N=9)$ is presented in Figure 5a. After solving the smallscale equations in separate sections and adding the large and small components according to Eq. (4), the resulting space charge distribution (Fig. 5b)

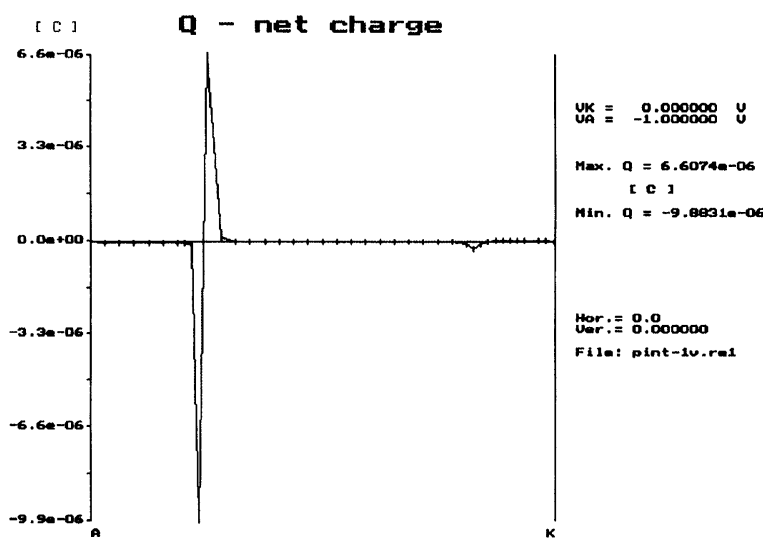

FIGURE 4 Net charge $\left(Q=p-n+N_{d}-N_{a}\right)$ for the reverse bias $V_{A K}=-1.0 \mathrm{~V}$, calculated with the traditional method on one non uniform discretization grid.

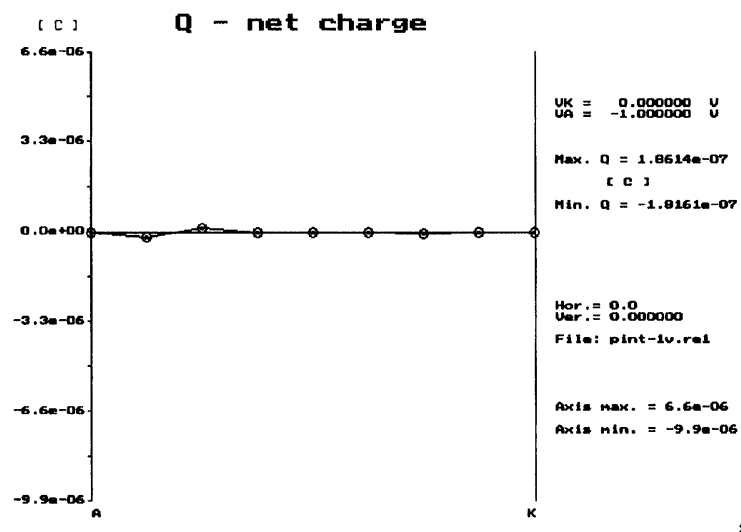

a)

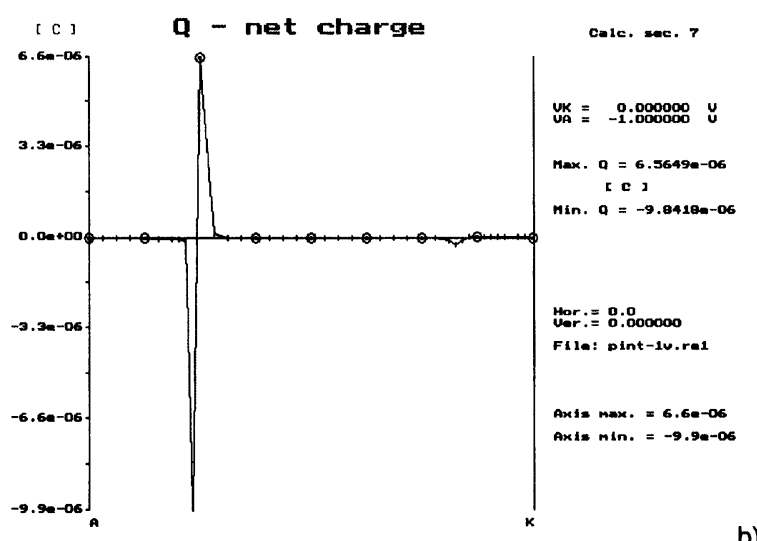

b)

FIGURE 5 Net charge distributions for the reverse bias $-1.0 \mathrm{~V}$ : a) results calculated using only the large-scale grid with 9 points uniformly distributed, b) results obtained using AD method after adding the large-scale and small-scale components (the thicker, circled grid lines correspond to the large-scale grid); note the good agreement with the reference results in Figure 4.

was almost identical to the reference space charge distribution obtained with the traditional method (Fig. 4). While the current density for the reverse bias calculated using only the coarse large-scale grid is the same order as the reference solution (error below $36 \%$ ), the local charge distribution is absolutely missing from large-scale solution (Fig. 5a), and is updated only by adding the small-scale components (Fig. 5b).

Here, we discuss if there is any physical meaning of the basically arithmetic operations leading to the additive decomposition of the semiconductor model into large-scale and small-scale compon- 
ents. The method was originally conceived for a more accurate description of turbulent fluid flow, where the two types of phenomena could be observed: large-scale fluid flow in the main stream, and turbulences which might be perceived as small-scale fluctuations over the large-scale flow. Is there anything similar in semiconductors? The results shown above suggest that the current density may be treated as a "large-scale value" as it can be calculated with a reasonable accuracy even on very coarse grids. We have checked various forward bias values; the error of largescale current density ranged from $0.8 \%$ for $V_{A K}=+1.0 \mathrm{~V}$ to $20 \%$ for $V_{A K}=+2.0 \mathrm{~V}$. In the 1-D case, the value of the total current density should be uniform and equal over the entire length of the device, which again shows its large-scale character. On the other hand, results in Figure 5 indicate that the space charge shows a "small-scale character", as it can be only calculated using a fine small-scale grid. These observations actually agree with the more rigorous analysis of the basic semiconductor equations using a singular perturbation approach [10]. There, it is concluded that if the Scharfetter-Gummel discretizetion [11] of the continuity equations is used (which is used also in our calculations), the current density can be calculated accurately even on a coarse discretization grid, virtually ignoring "layers", i.e., thin regions of rapid variations of the potential and the carrier densities, which physically correspond to junctions between differently doped regions of the device. However, to solve the continuity equations and Poisson's equation accurately in the neighborhood of junctions, a much finer grid is required. Therefore, the distributions of the potential and carrier densities (hence, also the net charge) indicate a "small-scale character" near the junctions. The presented additive decomposition approach revealed also this feature of the semiconductor drift-diffusion equations.

In both cases, i.e., forward bias and reverse bias of the $p$-i-n diode, the additive decomposition results agreed quite well with the results obtained with traditional one-grid approach, while the AD method allows for potentially significant reduction of matrix computation and computer memory requirements. Although in this case the sections with small-scale grid were calculated sequentially, the code has been written as if the sections were processed in parallel. Hence, the possible parallel implementation of $\mathrm{AD}$ has been verified. Extensive parallelization studies have been conducted for 1-D, 2-D and 3-D Navier-Stokes equations solved with this general approach by McDonough and co-workers [12-14].

\section{CONCLUSION}

The main advantage of the new method is that the matrix equations for sections with small-scale points can be solved independently, which allows for parallel computations and smaller memory requirements. Furthermore, computations for few large-scale points are very fast. After the largescale solution is computed an initial guess for the small-scale solution may be updated using interpolation of large-scale solution, which also leads to more efficient computation.

After successful implementation and testing of the AD method for the drift-diffusion equations, the authors intend to apply the new approach to the hydrodynamic semiconductor model, for which the AD technique should be also suitable.

\section{References}

[1] McDonough, J. M. and Bywater, R. J., "Large-Scale Effects on Local Small-Scale Chaotic Solutions to Burgers' Equation", AIAA Journal, 24(12), 1924-1930, December 1986.

[2] McDonough, J. M. and Bywater, R. J., "Turbulent Solutions from an Unaveraged, Additive Decomposition of Burgers' Equation", The Third Joint ASCE/ASME Mechanics Conference, University of California, San Diego, La Jolla, California, 7-11, July 9-12, 1989.

[3] McDonough, J. M. and Saito, K. (1994). "Local SmallScale Interaction of Turbulence with Chemical Reactions in $\mathrm{H}_{2}-\mathrm{O}_{2}$ Combustion", Fire Science and Technology, 14(1-2), 1-18.

[4] Selberherr, S. (1984). Analysis and Simulation of Semiconductor Devices, Vienna - New York: Springer-Verlag.

[5] Brown, R., Perry, P. and Shen, Z., "The Additive Decomposition for the Two-Dimensional Incompressible Navier-Stokes Equations: Convergence Theorems and 
error Estimates," accepted by SIAM Journal on Mathematical Analysis.

[6] Turowski, M., Grecki, M. and Napieralski, A., "PASSC - Package for 2-D Analysis of Semiconductor Structures in Circuits", XIII Symposium "Electromagnetic Phenomena in Nonlinear Circuits", Poznan, Poland, pp. 189-194, May 25-28, 1994.

[7] Turowski, M. and Napieralski, A., "Two-dimensional analysis of GTO switching under the influence of external circuit", IEE Proc.-Circuits, Devices and Systems, 141(6), 483-488, December 1994.

[8] Turowski, M. (1995). "Educational Software for the Analysis of the Behaviour of Power Semiconductor Devices", Chapter 10 in monographic book: Computers in Electrical Engineering Education - Research, Development and Application, pp. 153 - 165, Monash Engineering Education Series, EEERG-USICEE, Melbourne, Australia.

[9] Brauer, E. J., Turowski, M. and McDonough, J. M., "Additive Decomposition of the Drift-Diffusion Model", Fifth International Workshop on Computational Electronics, IWCE-5, Notre Dame, Indiana, USA, May 28-30, 1997.

[10] Markowich, P. A., Ringhofer, C. A., Selberherr, S. and Lentini, M. (1983). "A Singular Perturbation Approach for the Analysis of the Fundamental Semiconductor Equations", IEEE Trans. Electron Devices, ED-30(9), $1165-1180$.

[11] Scharfetter, D. L. and Gummel, H. K. (1969). "Large scale analysis of a silicon Read diode oscillator", IEEE Trans. Electron Devices, ED-16(1), 64-77.

[12] McDonough, J. M. and Wang, D. (1995). "Additive Turbulent Decomposition: A Highly Parallelizable Turbulence Simulation Technique", in Parallel Computational Fluid Dynamics, Satofuka et al. (eds.), Elsevier Science B.V., pp. $129-136$.

[13] Mukerji, S. and McDonough, J. M. (1996). "Parallel Computation of 3-D Small-Scale Turbulence Via Additive Turbulent Decomposition", in Parallel Computational Fluid Dynamics, Ecer et al. (eds.), Elsevier Science B.V., pp. $465-472$

[14] McDonough, J. M. (1997). "Parallelization of ATD/ Chaotic Map Turbulence Models", in Parallel Computational Fluid Dynamics, Schiano et al. (eds.), Elsevier Science B.V., pp. $92-99$.

\section{Authors' Biographies}

Elizabeth J. Brauer received the Ph.D. degree from University of Illinois at Urbana-Champaign. She worked at Motorola, Inc., National Semiconduc- tor, Inc. and the University of Kentucky. Presently, she is the Director of the Advanced Microelectronics Laboratory in the Department of Electrical Engineering at Northern Arizona University in Flagstaff, AZ. Her research interests involve $\mathrm{CAD}$, verification, and testing of integrated circuits, modeling of semiconductor devices, and neural networks.

Marek Turowski received the M.Sc. and Ph.D. degrees from the Technical University of Lodz, Poland, in 1983 and 1992, respectively. Since 1983 he has been with the Technical University of Lodz. Currently he is a post-doc scholar at the University of Kentucky in Lexington, USA. In 1992/93 he was for one year at the University of Sydney, Australia. His research interests include numerical methods for 2-D and 3-D modeling and simulation of semiconductor devices, electromagnetic fields, and thermal phenomena in electronic devices. He is author or co-author of over 50 papers in international journals and conference proceedings.

James M. McDonough is an Associate Professor in the Department of Mechanical Engineering at the University of Kentucky. He received a BS in Aero-Astro Engineering from the Ohio State University (1968) and MA, Applied Mathematics (1975) and Ph.D., Engineering (1980) degrees from the University of California, Los Angeles. $\mathrm{He}$ has nearly 15 years of industrial experience, primarily in the aerospace industry, in addition to his academic experience. His research interests span a broad spectrum of topics in numerical computation, CFD and nonlinear chaotic dynamical systems. He has published more than 60 papers in archival journals and conference proceedings. 

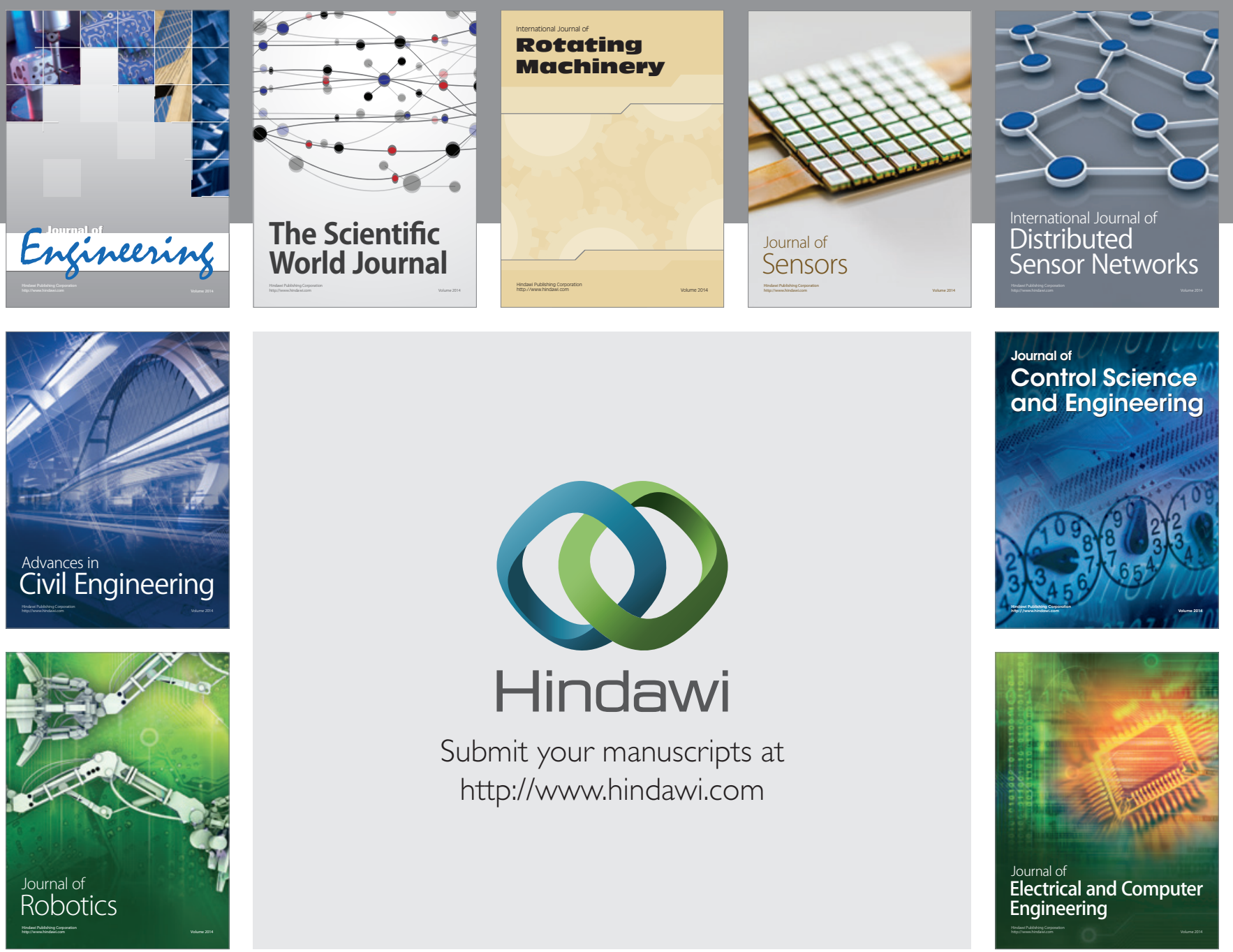

Submit your manuscripts at

http://www.hindawi.com
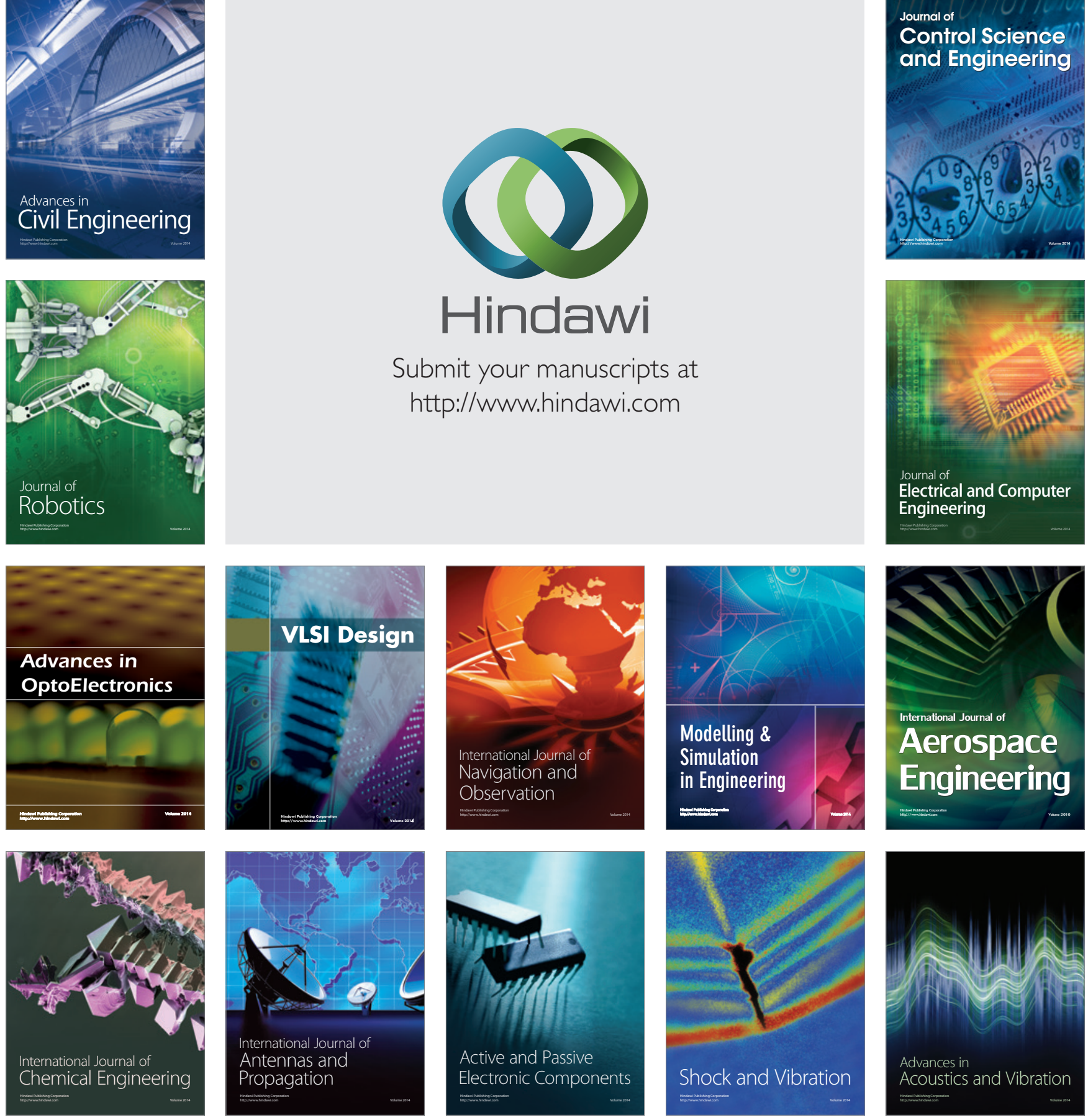